\title{
Cirurgia de revascularização do miocárdio: características da internação e alterações relacionadas ao tempo de internação
}

\author{
Cintia Koerich ${ }^{1}$, Gabriela Marcellino de Melo Lanzoni ${ }^{2}$, Giovana Dorneles Callegaro Higashi ${ }^{3}$, \\ Alacoque Lorenzini Erdmann ${ }^{4}$, Betina Hörner Schlindwein Meirelles ${ }^{5}$, Maria Aparecida Baggio ${ }^{6}$
}

\author{
${ }^{1}$ Enfermeira, Mestre em Enfermagem. \\ Discente do Programa de Pós-Graduação \\ em Enfermagem da Universidade Federal \\ de Santa Catarina, nível Doutorado. \\ Enfermeiro do Instituto de Cardiologia de \\ Santa Catarina 2. São José, SC, Brasil. E- \\ mail: cintia.koerich@gmail.com. \\ ${ }^{2}$ Enfermeira, Doutora em Enfermagem. \\ Professora Adjunta da Universidade \\ Federal de Santa Catarina. Florianópolis, \\ SC, Brasil. E-mail: gabimrc@gmail.com. \\ ${ }^{3}$ Enfermeira, Doutora em Enfermagem. \\ Professora Substituta da Universidade \\ Federal de Santa Catarina. Florianópolis, \\ SC, Brasil. E-mail: \\ gio.enfermagem@gmail.com. \\ ${ }^{4}$ Enfermeira, Doutora em Filosofia de \\ Enfermagem. Professora Titular da \\ Universidade Federal de Santa Catarina. \\ Florianópolis, SC, Brasil. E-mail: \\ alacoque@newsite.com.br. \\ ${ }^{5}$ Enfermeira, Doutora em Enfermagem. \\ Professora Associada da Universidade \\ Federal de Santa Catarina. Florianópolis, \\ SC, Brasil. E-mail: betina.hsm@ufsc.br. \\ ${ }^{6}$ Enfermeira, Doutora em Enfermagem. \\ Docente do Ensino Superior da \\ Universidade Estadual do Oeste do Paraná. \\ Cascavel, PR, Brasil. E-mail: \\ mariabaggio@yahoo.com.br.
}

Recebido: 22/08/2016.

Aceito: 11/09/2017.

Publicado: 31/12/2017.

Como citar esse artigo: Koerich C, Lanzoni GMM, Higashi GDC, Erdmann AL, Meirelles BHS, Baggio MA. Cirurgia de revascularização do miocárdio: características da internação e alterações relacionadas ao tempo de internação. Rev. Eletr. Enf. [Internet]. 2017 [acesso em: ____;19:a45. Disponível em: http://doi.org/10.5216/ree.v19.42870.

\section{RESUMO}

O estudo teve como objetivo identificar as características da internação e alterações apresentadas por indivíduos submetidos à Cirurgia de Revascularização do Miocárdio e sua associação com tempo de internação para cirurgia. Estudo epidemiológico observacional e transversal, realizado entre março de 2013 a março de 2014, com 99 indivíduos submetidos à Cirurgia de Revascularização do Miocárdio. Verificou-se que dor no peito e angina/dor torácica identificados no pré-operatório e insuficiência respiratória, hipertermia, Hipertensão Arterial Sistêmica e arritmias apresentadas no pós-operatório foram preditores para maior tempo de internação. A associação entre as características da internação e as alterações clínicas com o tempo de Internação de indivíduos submetidos à Cirurgia de Revascularização do Miocárdio fornecem subsídios para atuação do enfermeiro e demais profissionais de saúde e gestores, a fim de criar estratégias para detecção precoce de intercorrências relacionadas à Cirurgia de Revascularização do Miocárdio.

Descritores: Tempo de Internação; Doenças Cardiovasculares; Cirurgia Torácica; Cuidados de Enfermagem; Gestão em Saúde.

\section{INTRODUÇÃO}

Estima-se que as doenças cardiovasculares (DCV) sejam, no mundo, a principal causa de morte e perda da qualidade de vida relacionada a incapacidades, e incluída neste grupo a Doença Arterial Coronariana (DAC), em especial o Infarto agudo do miocárdio (IAM) será responsável nos próximos anos por aproximadamente uma morte por minuto ${ }^{(1)}$. As DCV estão incluídas nas doenças do aparelho 
circulatório e são caracterizadas como doença crônica não transmissível ${ }^{(2)}$. No Brasil, as doenças do aparelho circulatório representaram a terceira causa de hospitalizações pelo Sistema Único de Saúde (SUS) no ano de 2015, com 1.124.156 internações, sendo a insuficiência cardíaca a causa mais frequente ${ }^{(3)}$.

A internação hospitalar é necessária de acordo com a gravidade e o estágio avançado da DAC, a qual exigirá tratamento clínico ou cirúrgico ${ }^{(4)}$. Nos casos em que a intervenção coronária percutânea é insuficiente, a Cirurgia de Revascularização do Miocárdio (CRM) tem sido realizada com regularidade no SUS, no entanto, apesar dos grandes benefícios alcançados, as intercorrências pós-operatórias costumam ser comuns, tais como, atelectasia, pneumonia, broncoespasmo, insuficiência respiratória, dentre outras ${ }^{(5)}$.

O aumento da demanda de CRM no SUS contribui para o aumento do tempo de espera pela cirurgia, propiciando o evento de intercorrências e o agravamento do quadro clínico dos indivíduos que aguardam a cirurgia ${ }^{(6)}$. Torna-se importante conhecer quais intercorrências apresentadas pelo paciente no pré e pósoperatório de CRM estão relacionadas ao período de internação para cirurgia, visto que o tempo de internação prolongado tende a estar diretamente relacionado a alterações clínicas no paciente, aumentando os custos da cirurgia cardíaca no SUS ${ }^{(7)}$.

Neste sentido, os profissionais de enfermagem e de saúde possuem importante atuação no cenário de cuidados ao paciente acometido por doença cardiovascular, em especial por doença arterial coronariana, principalmente devido à instabilidade hemodinâmica e às potenciais complicações da doença. Considerar a realidade vivenciada por esses profissionais é imprescindível para o planejamento de ações de gestão do cuidado ao paciente submetido à CRM. A atuação do enfermeiro se destaca por meio da prática assistencial responsável, considerando a maior proximidade ao paciente durante o processo cirúrgico ${ }^{(8)}$, que envolve desde a indicação cirúrgica até após a alta hospitalar, incluindo a reabilitação.

Portanto, o objetivo deste estudo é identificar as características da internação e alterações apresentadas por indivíduos submetidos à Cirurgia de Revascularização do Miocárdio e sua associação com tempo de internação para cirurgia.

\section{MÉTODO}

Estudo epidemiológico observacional e transversal. O cenário do estudo foi uma instituição de saúde pública hospitalar de referência cardiovascular no sul do Brasil, situada em Santa Catarina. A instituição possui 109 leitos de internação, sendo 79 nas unidades de internação médica cirúrgica (UI), 10 leitos na Unidade de Terapia Intensiva (UTI) e 20 leitos na emergência, onde funcionam leitos de cuidados semiintensivos e de observação.

A população foi constituída de todos os indivíduos internados na instituição, durante o período de março de 2013 a março de 2014. O critério de inclusão foi estar internado na instituição e ter realizado a CRM. Aqueles que não apresentavam condições clínicas favoráveis para responder a entrevista (seja por desorientação e/ou dislalia), ou ocorrido o óbito antes da realização do procedimento cirúrgico, foram excluídos do estudo. No total, 110 internações foram reguladas para CRM, destes 11 estavam entre os 
critérios de exclusão.

Os objetivos do estudo foram apresentados aos participantes, e aqueles que concordaram em participar assinaram o termo de consentimento livre e esclarecido. Foi realizado contato diário com os participantes da pesquisa durante o período de internação a fim de detectar alterações no quadro de saúde. Informações sobre o diagnóstico e quadro clínico de desconhecimento dos participantes foram coletadas em prontuário com a devida autorização dos participantes.

Os dados foram coletados por meio de um roteiro de entrevista estruturado, o qual recebeu a contribuição de dois profissionais atuantes na instituição para sua elaboração, sendo estes um médico e um enfermeiro com experiência na área. No momento da aplicação, os dados coletados foram diretamente digitados em uma planilha eletrônica do programa Excel ${ }^{\circ}$ da Microsoft, e ao final, exportados para o Software SPSS Statistics versão 22.0 para a realização da análise estatística.

$\mathrm{Na}$ análise descritiva foram verificadas as frequências e os percentuais para as medidas qualitativas, e as médias e os desvios padrão para as medidas quantitativas, sendo considerados os seguintes agrupamentos e variáveis:

- Características da internação: período de internação (Tempo de internação; Tempo de préoperatório; Tempo de pós-operatório) e elementos do perioperatório (Possui acompanhante antes da cirurgia; Período que fica com acompanhante; Possui acompanhante depois da cirurgia; Teve cirurgia suspensa; Motivo da suspensão cirúrgica; Recebeu orientações pré-operatórias; De que profissional recebeu orientação)

- Alterações clínicas: sintomas apresentados no pré-operatório (Ansiedade; Fraqueza; Tontura; Náuseas/vômitos; Palpitações; Dor no peito; Falta de ar, febre); Intercorrências apresentadas no préoperatório (Angina/ Dor Torácica; Hipertensão Arterial Sistêmica (HAS); Hipotensão arterial; Taquicardia; Bradicardia; Arritmias; Taquipnéia; Bradipnéia; Dispnéia; Acidente Vascular Cerebral (AVC); Hipertemia); Intercorrências apresentadas no pós-operatório imediato-UTI (Insuficiência Renal; Insuficiência Respiratória; AVC; Arritmias (Fibrilação Atrial - FA, Bloqueio Átrio Ventricular Total - BAVT); Infarto Agudo do Miocárdio (IAM) transoperatório; Hemorragia/Alterações Células Sanguíneas; Infecções); Intercorrências apresentadas no pós-operatório mediato-UI (Angina/ Dor Torácica; HAS; Hipotensão arterial; Taquicardia; Bradicardia; Arritmias; Taquipneia; Bradipneia; Dispneia; AVC; Hipertemia). Os sintomas referem-se às queixas dos participantes durante o acompanhamento dos pesquisadores e as intercorrências ao registro dos profissionais de saúde no prontuário do paciente.

Para as medidas de atributo foi utilizado o teste exato de Fisher e para as medidas contínuas o teste $t$ Student ou o teste não-paramétrico de Kruskal-Wallis quando o teste t não pode ser aplicado. Em relação aos testes de comparação, considerou-se como variáveis de desfecho às referente à categoria "Período de Internação" e como variáveis independentes aquelas referentes às categorias: elementos do perioperatório, Sintomas apresentados no pré-operatório, Intercorrências apresentadas no pré-operatório, Intercorrências 
apresentadas no pós-operatório imediato (UTI) e Intercorrências apresentadas no pós-operatório mediato (UI).

Para os testes estatísticos foi assumido um nível de confiança de $95 \%$ e um erro amostral de $5 \%$.

A pesquisa foi aprovada pelo Comitê de Ética em Pesquisa com Seres Humanos da Universidade Federal de Santa Catarina (CEPSH/USFC) sob o número 120.184 em 2012, estando de acordo com a Resolução n. 466/12 do Conselho Nacional de Saúde.

\section{RESULTADOS}

A caracterização sociodemográfica mostra que $70,7 \%$ dos participantes são homens, $61 \%$ casados, $53,5 \%$ aposentados, e média de idade de 61,3 anos ( $D P$ 8,5). Na análise descritiva a média do tempo de internação ficou em 40,3 (DP 19,4) dias, enquanto a média do tempo de pré-operatório ficou em 22,8 (DP $16,1)$ dias, já a média do tempo de pós-operatório ficou em $10,9(D P 10,2)$ dias.

Quanto aos elementos do perioperatório, $14,1 \%(n=14)$ dos participantes possuíam acompanhantes, destes nove em período integral. Após a cirurgia, 59,5\% $(n=59)$ declararam ter acompanhante, destes $47,5 \%$ ( $n=28)$ foram acompanhados por filhos, 38,9\% $(n=23)$ por cônjuge e 13,5\% $(n=8)$ por outros.

Dos 99 participantes, 12,1\% ( $n=12$ ) tiveram a cirurgia suspensa, mas realizaram posteriormente a CRM, entre os motivos $50 \%$ ( $n=6)$ foi substituída por cirurgia de urgência para outro paciente, $41,6 \%(n=5)$ por falta de leito de UTI e 8,3\% ( $n=1)$ por falta de reserva de sangue. Ainda, 47,4\% $(n=47)$ afirmaram ter recebido orientações pré-operatórias, 59,6\% ( $n=28)$ destes pela equipe multiprofissional, 31,9\% $(n=15)$ pelo médico e $8,5 \%(n=4)$ pelo enfermeiro.

Dos sintomas mais frequentes no período pré-operatório, $76,7 \% \quad(n=76)$ dos participantes apresentaram ansiedade, $36,3 \%(n=36)$ dor no peito e $23,2 \%(n=23)$ falta de ar. Outros sintomas representaram menos de $10 \%$. Quanto às intercorrências no período pré-operatório, 40,4\% ( $n=40)$ apresentaram angina/dor torácica, 17,1\% ( $n=17)$ HAS e 15,1\% ( $n=15)$ dispnéia. Outras intercorrências representaram menos de $10 \%$.

Quanto as intercorrências mais frequentes no período pós-operatório imediato (UTI), 21,2\% ( $n=21$ ) apresentaram Hemorragia/Alterações das células sanguíneas, 17,1\% ( $n=17)$ arritmias (FA/BAVT) e quanto às complicações pulmonares 21,2\% ( $n=21)$ tiveram broncoespasmo e atelectasia, e $11,1 \%$ insuficiência respiratória, outras intercorrências representaram menos de 10\%. No período pós-operatório mediato (UI), entre as intercorrências mais prevalentes destacam-se broncoespasmo, atelectasia (23,2\%; n=23), além de pneumonia/broncopneumonia $(2,0 \% ; n=2)$ e dispneia $(12,1 \% ; n=12)$, outras intercorrências representaram menos de $10 \%$.

$\mathrm{Na}$ análise comparativa, do agrupamento Características da internação, as variáveis da categoria "elementos perioperatórios" não apresentaram diferença significativa para as variáveis da categoria "período de internação" ( $p>0,05)$.

Do agrupamento Alterações clínicas, na categoria "Sintomas apresentados no pré-operatório" a 
variável dor no peito $(p=0,013)$, apresentou diferença significativa para a variável Tempo de internação da categoria "período de internação", assim como, a variável angina/dor torácica $(p=0,024)$, da categoria "Intercorrências apresentadas no pré-operatório". Em ambos os casos o tempo de internação foi maior. As demais variáveis das categorias supracitadas não apresentaram diferença significativa em relação às variáveis da categoria "período de internação".

Em relação de as categorias "Período internação" e "Intercorrências apresentadas no pós-operatório imediato (UTI)", a variável insuficiência respiratória apresentou diferença significativa para a variável tempo de pós-operatório $(p=0,017)$ e tempo de internação $(p=0,039)$. As demais variáveis da categoria "Intercorrências apresentadas no pós-operatório imediato (UTI)" não apresentaram diferença significativa em relação às variáveis da categoria "período de internação".

Quanto as categorias "Período de internação" e "Intercorrências apresentadas no pós-operatório mediato (UI)", as variáveis arritmias $(p=0,047)$ e hipertermia $(p=0,006)$ apresentaram diferença significativa para a variável tempo de internação. As variáveis $\operatorname{HAS}(p=0,048)$ e a arritmia $(p=0,001)$ apresentaram diferença significativa para a variável tempo de pós-operatório. As demais variáveis da categoria "Intercorrências apresentadas no pós-operatório mediato (UI)" não apresentaram diferença significativa em relação às variáveis da categoria "período de internação".

Sobre as variáveis que compõem o agrupamento das Alterações clínicas, a Tabela 1 apresenta somente aquelas preditoras para maior tempo de internação ou maior tempo de pós-operatório entre os indivíduos submetidos à CRM. 
Tabela 1: Comparação entre alterações clínicas e período de internação em 99 pacientes submetidos à Cirurgia de Revascularização do Miocárdio, Santa Catarina, Brasil, 2014.

\begin{tabular}{|c|c|c|}
\hline \multirow{2}{*}{ Variáveis } & \multicolumn{2}{|c|}{ Tempo de internação (dias) } \\
\hline & Média/DP & p-valor \\
\hline \multicolumn{3}{|l|}{ Sintoma pré-operatório: Dor no peito } \\
\hline Com & $47,1(22,6)$ & 0,013 \\
\hline Sem & $36,5(16,3)$ & \\
\hline \multicolumn{3}{|l|}{ Intercorrência pré-operatória: Angina/dor torácica } \\
\hline Com & $46,1(22,1)$ & 0,024 \\
\hline Sem & $36,4(16,5)$ & \\
\hline \multicolumn{3}{|c|}{ Intercorrência no pós-operatório imediato (UTI): Insuficiência Respiratória } \\
\hline Com & $56,1(27,9)$ & 0,038 \\
\hline Sem & $38,4(17,3)$ & \\
\hline \multicolumn{3}{|l|}{ Intercorrência no pós-operatório mediato (UI): Arritmia } \\
\hline Com & $59,2(23,6)$ & 0,047 \\
\hline Sem & $39,3(18,8)$ & \\
\hline \multicolumn{3}{|c|}{ Intercorrência no pós-operatório mediato (UI): Hipertermia } \\
\hline Com & $73,0(21,9)$ & 0,006 \\
\hline Sem & $39,0(18,2)$ & \\
\hline \multirow{2}{*}{ Variáveis } & \multicolumn{2}{|c|}{ Tempo de pós-operatório } \\
\hline & Média/DP & p-valor \\
\hline \multicolumn{3}{|c|}{ Intercorrência no Pós-operatório Imediato (UTI): Insuficiência Respiratória } \\
\hline Com & $23,9(23,6)$ & 0,017 \\
\hline Sem & $9,3(5,5)$ & \\
\hline \multicolumn{3}{|c|}{ Intercorrência no pós-operatório mediato (UI): Hipertensão Arterial Sistêmica } \\
\hline Com & $14,6(8,6)$ & 0,048 \\
\hline Sem & $10,6(10,3)$ & \\
\hline \multicolumn{3}{|l|}{ Intercorrência no pós-operatório mediato (UI): Arritmia } \\
\hline Com & $30,2(24,5)$ & 0,001 \\
\hline Sem & $9,9(8,0)$ & \\
\hline
\end{tabular}

Testes estatísticos: teste -Student ou teste não-paramétrico de Kruskal-Wallis.

\section{DISCUSSÃO}

A média do tempo de internação em dias neste estudo foi de 40,3 (DP 19,4) dias, corroborando com estudo realizado na mesma instituição no ano de 2011, que buscou traçar o perfil clínico-epidemiológico e cirúrgico dos pacientes submetidos à CRM, com uma mediana de 35,5 dias $^{(6)}$. Já a média do tempo de pósoperatório foi de em 10,9 dias (DP 10,2) dias, sendo três vezes maior que o achado em estudo realizado no Rio Grande do Sul, em que a média de tempo de internação pós-operatória foi de 3,77 (DP 3,53) dias ${ }^{(9)}$.

Com relação a média de tempo de pós-operatório, estudo realizado em Omã, país árabe localizado na costa sudeste da Península Arábica, 30,5\% dos pacientes submetidos à cirurgia cardíaca em um grande hospital de referência, entre 2009 e 2013, tiveram o tempo de pós-operatório prolongado ( $\geq 11$ dias), cujo principal determinante foi o número de intercorrências ${ }^{(10)}$.

Outras investigações trazem a média de tempo de internação para CRM entre três e 11 dias e associam a baixa permanência hospitalar à redução de complicações e riscos relacionados à internação(11-12), influenciando inclusive a disponibilidades de leitos ${ }^{(11)}$.

Apesar de não apresentar diferença significativa para o tempo de internação neste estudo, a ansiedade foi um sintoma pré-operatório relatado por 76 (76,7\%) participantes e, apenas 14 (14,1\%) participantes 
informaram ter acompanhante no pré-operatório. Em outro estudo, que buscou comparar a intensidade de sintomas de ansiedade em pessoas no pré-operatório de cirurgia cardíaca, confrontou três grupos, aqueles que receberam o acolhimento de familiar, outro somente o acolhimento do enfermeiro e um grupo que não recebeu acolhimento. Observou-se que a redução dos sintomas de ansiedade foi maior no grupo que recebeu acolhimento dos familiares em relação aos outros dois grupos, sugerindo que a presença do familiar no préoperatório pode contribuir para a redução de sintomas de ansiedade no pré-operatório de cirurgias cardíacas $^{(13)}$. A ansiedade pode refletir negativamente na recuperação pós-operatória por acarretar alterações fisiológicas ${ }^{(14)}$ sendo evidenciada sua redução em pacientes que recebem orientações préoperatórias $^{(15)}$.

A angina/dor torácica foi a alteração de maior frequência no pré-operatório de CRM. A presença desta alteração no pré-operatório esteve relacionada ao maior tempo de internação $(p=0,0242)$. Estudo que analisou a presença de angina instável em pacientes no pré-operatório de CRM identificou que estes pacientes utilizaram mais medicações durante a internação, monitorização com Swan-Ganz e suporte com balão intra-aórtico do que aqueles sem angina instável, necessitando assim de maior tempo de internação ${ }^{(16)}$.

Além da angina, a HAS aparece como uma alteração frequente no pré-operatório e pós-operatório mediato e, neste último caso, foi associada estatisticamente a maior tempo de pós-operatório $(p=0,0487)$. Estudo realizado no sudeste do Brasil identificou que a HAS esteve diretamente relacionada com lesões em órgãos vitais de pacientes submetidos a CRM, caracterizando maior gravidade clínica e consequente maior probabilidade de piores desfechos ${ }^{(17)}$.

O tempo prolongado de internação apresentou associação estatística com insuficiência respiratória no pós-operatório imediato na UTI $(p=0,017)$ e no pós-operatório mediato na UI com a presença de arritmia (FA/BAVT) $(p=0,047)$ e hipertermia $(p=0,006)$. As intercorrências pós-operatórias mais frequentes na UTI foram as hemorragias e complicações pulmonares. Já na UI prevaleceram as complicações pulmonares.

Estudo realizado no sudeste do Brasil analisou as intercorrências pós-operatórias de CRM e identificou que a mais frequente foi a hemorragia ou baixo débito pós-procedimento, o que ocorreu em de $50 \%$ dos casos, sendo que $60 \%$ dos pacientes que foram a óbito após a CRM apresentaram cinco ou mais complicações no pós-operatório ${ }^{(18)}$. Outro estudo avaliou as principais alterações apresentadas por pacientes idosos após CRM, estando entre elas as complicações respiratórias, pneumonia e reintervenção por sangramento ${ }^{(19)}$.

Em relação à insuficiência respiratória, pode-se afirmar que pacientes submetidos à CRM costumam desenvolver disfunção pulmonar pós-operatória ${ }^{(12,19)}$ com redução importante dos volumes pulmonares, prejuízos na mecânica respiratória, diminuição na complacência pulmonar e aumento do trabalho respiratório, o que acaba aumentando o tempo de internação. Neste caso, a fisioterapia respiratória pode ser parte integrante na gestão do cuidado ao paciente acometido por doença cardiovascular, tanto no pré quanto no pós-operatório de $\mathrm{CRM}^{(5,20)}$.

As arritmias, por sua vez, são consideradas alterações pós-operatórias frequentes quando se trata de cirurgia cardíaca, em especial a fibrilação atrial ${ }^{(12,19)}$. Em estudo realizado no sul do Brasil, as intercorrências 
pós-operatórias ocorreram em 48,3\% da amostra, sendo a mais prevalente a fibrilação atrial, com $20,7 \%$, sendo que o impacto da FA no pós-operatório de CRM foi diretamente relacionado ao aumento do tempo de internação $(p<0,001)^{(21)}$. Outro estudo apontou que $208(8,15 \%)$ pacientes apresentaram bloqueio atrioventricular no pós-operatório de CRM, necessitando de estimulação cardíaca artificial temporária e de maior tempo de internação(22).

A hipertermia é uma das principais manifestações de quadro infeccioso e este está intimamente relacionado a complicações e óbito por choque séptico após CRM. Intercorrências do sistema respiratório, como a pneumonia e broncopneumonia, por exemplo, normalmente são infecções que acabam aumentando o tempo de internação após CRM $^{(23)}$.

Ao analisar os desfechos clínicos dos pacientes submetidos à cirurgia cardíaca ao sul do Brasil, estudo apontou a importância dos profissionais de saúde conhecerem as principais alterações no pós-operatório de cirurgia cardíaca. Em especial, a enfermagem deve reconhecer no paciente características de risco para intercorrências a fim de direcionar o cuidado às evidências que possam trazer danos ao paciente e assim consequentemente qualificar a assistência de enfermagem ${ }^{(9)}$.

Neste sentido, a disponibilidade e utilização da sistematização da assistência de enfermagem (SAE) e protocolos institucionais podem ser empregados pelo enfermeiro a fim de detectar precocemente alterações clínicas no paciente com indicação ou submetido à CRM otimizando o fluxo desses pacientes para a cirurgia, diminuindo o tempo de internação e consequentemente os custos para ao sistema de saúde. Conhecer as principais alterações destes pacientes a partir de ferramentas próprias facilita tal identificação e auxilia o enfermeiro na decisão terapêutica e no planejamento da assistência de enfermagem ${ }^{(24)}$.

\section{CONCLUSÃO}

Os resultados revelam as principais características da internação e alterações apresentadas por indivíduos no pré e pós-operatório de CRM, sendo estas: ansiedade, dor no peito, dor torácica, angina, falta de ar, dispnéia, Hipertensão Arterial Sistêmica, hemorragia, complicações pulmonares e arritmias. As variáveis do pré-operatório: dor no peito, dor torácica e angina e as variáveis do pós-operatório: insuficiência respiratória, hipertermia e arritmia foram associadas ao maior tempo de internação geral, como também se observou maior tempo de pós-operatório relacionado às variáveis do pós-operatório: insuficiência respiratória, HAS e arritmias.

Tais achados fornecem subsídios para atuação do enfermeiro, demais profissionais de saúde e gestores, a fim de criar estratégias de prevenção e detecção precoce de alterações relacionadas à DCV e a CRM que possam otimizar o tratamento e reduzir o tempo de internação prevenindo piores desfechos e reduzindo custos para o sistema de saúde. 
1. DATASUS [Internet]. Brasília: Ministério da Saúde; 2016 [acesso em: 30 dez. 2017]. Disponível em:

http://www.datasus.gov.br.

2. Stip MAC. A gerência do cuidado na enfermagem cardiovascular. Esc Anna Nery [Internet]. 2012 [acesso em: 30 dez. 2017];16(1):7-9. Disponível em: http://dx.doi.org/10.1590/S1414-81452012000100001.

3. Roger VL, Go AS, Lloyd-Jones DM, Benjamin EJ, Berry JD, Borden WB, et al. Executive Summary: Heart Disease and Stroke Statistics--2012 Update: A Report From the American Heart Association. Circulation [Internet]. 2012 [acesso em: 30 dez. 2017];125(1):188-97. Disponível em: https://doi.org/10.1161/CIR.0b013e3182456d46.

4. Erdmann AL, Lanzoni GMM, Callegaro GD, Baggio MA, Koerich C. Understanding the process of living as signified by myocardial revascularization surgery patients. Rev Lat Am Enfermagem [Internet]. 2013 [acesso em: 30 dez.

2017];21(1):332-9. Disponível em: http://dx.doi.org/10.1590/S0104-11692013000100007.

5. Cavenaghi S, Ferreira LL, Marino LHC, Lamari NM. Fisioterapia respiratória no pré e pós-operatório de cirurgia de revascularização do miocárdio. Rev Bras Cir Cardiovasc [Internet]. 2011 [acesso em: 30 dez. 2017];26(3):455-61. Disponível em: http://dx.doi.org/10.5935/1678-9741.20110022.

6. Tonial R, Moreira DM. Perfil clínico-epidemiológico dos pacientes submetidos à cirurgia de revascularização do miocárdio no instituto de cardiologia de Santa Catarina, São José - SC. Arquivos Catarinenses de Medicina [Internet]. 2011 [acesso em: 30 dez. 2017];40(4) 42-6. Disponível em: http://www.acm.org.br/revista/pdf/artigos/894.pdf. 7. Titinger DP, Lisboa LAF, Matrangolo BLR, Dallan LRP, Dallan LAO, Trindade EM, et al. Cardiac Surgery Costs According to the Preoperative Risk in the Brazilian Public Health System. Arq Bras Cardiol [Internet]. 2015 [acesso em: 30 dez. 2017];105(2):130-8. Disponível em: http://dx.doi.org/10.5935/abc.20150068.

8. Lanzoni GMM, Higashi GDC, Koerich C, Erdmann AL, Baggio MA. Factors which influence the process of experiencing cardiac revascularization. Texto Context - Enferm [Internet]. 2015 [acesso em: 30 dez. 2017];24(1):270-8. Disponível em: http://dx.doi.org/10.1590/0104-07072015003760012.

9. Santos MBK, Silveira CR, Moraes MAP, Souza EN. Desfechos clínicos de pacientes submetidos à cirurgia cardíaca em um hospital do noroeste do Rio Grande do Sul. Revista de Enfermagem da UFSM [Internet]. 2016 [acesso em: 30 dez. 2017];6(1):102-11. Disponível em: http://dx.doi.org/10.5902/2179769216467.

10. Almashrafi A, Alsabti H, Mukaddirov M, Balan B, Aylin P. Factors associated with prolonged length of stay following cardiac surgery in a major referral hospital in Oman: a retrospective observational study. BMJ Open [Internet]. 2016 [acesso em: 30 dez. 2017];6(6):e010764. Disponível em: http://doi.org/10.1136/bmjopen-2015-010764.

11. Ponte K, Silva L, Borges M, Aragão A, Arruda L, Galiza F. Characterization of people in cardiac surgeries: a descriptive study. Revista de Pesquisa: Cuidado é Fundamental Online [Internet]. 2013 [acesso em: 30 dez. 2017];5(2):3677-83. Disponível em: http://doi.org/10.9789/2175-5361.2013v5n2p3677.

12. Sousa AG, Fichino MZS, Silva GS, Bastos FCC, Piotto RF. Epidemiology of coronary artery bypass grafting at the Hospital Beneficência Portuguesa, São Paulo. Braz J Cardiovasc Surg [Internet]. 2015 [acesso em: 30 dez.

2017];30(1):33-9. Disponível em: http://dx.doi.org/10.5935/1678-9741.20140062.

13. Assis CC, Lopes JL, Nogueira-Martins LA, Barros ALBL. Acolhimento e sintomas de ansiedade em pacientes no préoperatório de cirurgia cardíaca. Rev. bras. enferm. [Internet]. 2014 [acesso em: 30 dez. 2017];67(3):401-7. Disponível em: http://dx.doi.org/10.5935/0034-7167.20140053.

14. Tully PJ, Bennetts JS, Baker RA, McGavigan AD, Turnbull DA, Winefield HR. Anxiety, depression, and stress as risk factors for atrial fibrillation after cardiac surgery. Heart Lung [Internet]. 2011 [acesso em: 30 dez. 2017];40(1):4-11. Disponível em: http://doi.org/10.1016/j.hrtlng.2009.12.010.

15. Teixeira MV, Corrêa AR, Silqueira SMF, Carvalho DV. Avaliação dos resultados das orientações pré-operatórias a pacientes submetidos à cirurgia cardíaca eletiva. Revista de Enfermagem do Centro Oeste Mineiro [Internet]. 2013 [acesso em: 30 dez. 2017];3(2):620-31. Disponível em:

http://www.seer.ufsj.edu.br/index.php/recom/article/view/329.

16. Sussenbach CP, Guaragna JC, Castagnino RS, Piccoli J, Albuquerque LC, Goldani MA, et al. Unstable angina does not increase mortality in coronary artery bypass graft surgery. Rev Bras Cir Cardiovasc [Internet]. 2013 [acesso em: 30 dez. 2017];28(3):391-400. Disponível em: http://dx.doi.org/10.5935/1678-9741.20130060.

17. Colósimo FC, Sousa AG, Silva GS, Piotto RF, Pierin AMG. Arterial hypertension and associated factors in patients submitted to myocardial revascularization. Rev. esc. enferm. USP [Internet]. 2015 [acesso em: 30 dez.

2017];49(2):201-7. Disponível em: http://dx.doi.org/10.1590/s0080-623420150000200003. 
18. Oliveira TML, Oliveira GMM, Klein CH, Silva NAS, Godoy PH. Mortality and complications of coronary artery bypass grafting in Rio de Janeiro, from 1999 to 2003. Arq Bras Cardiol [Internet]. 2010 [acesso em: 30 dez. 2017];95(3):303-

12. Disponível em: http://dx.doi.org/10.1590/S0066-782X2010005000091.

19. Lima VR, Leal CCG, Flávio AGC. Complicações pós-operatórias em idosos submetidos à revascularização do miocárdio. CuidArte Enfermafem [Internet]. 2014 [acesso em: 30 dez. 2017]; 8(1):48-54. Disponível em:

http://fundacaopadrealbino.org.br/facfipa/ner/pdf/cuidarte_enfermagem_v8_n1_jan_jun_2014.pdf.

20. Snowdon D, Haines TP, Skinner EH. Preoperative intervention reduces postoperative pulmonary complications but not length of stay in cardiac surgical patients: a systematic review. J Physiother [Internet]. 2014 [acesso em: 30 dez. 2017];60(2):66-77. Disponível em: http://dx.doi.org/10.1016/j.jphys.2014.04.002.

21. Sobral MLP, Barbieri LR, Gerônimo GMS, Trompieri DF, Souza TA, Rocca JMG, et al. O impacto da fibrilação atrial no pós-operatório de revascularização do miocárdio. Revista Latino-Americana de Marcapasso e Arritmia [Internet]. 2013 [acesso em: 30 dez. 2017];26(3):137-43. Disponível em: http://www.relampa.org.br/detalhe_artigo.asp?id=901. 22. Piantá RM, Ferrari ADL, Heck AA, Ferreira DK, Piccoli JCE, Albuquerque LC, et al. Atrioventricular block in coronary artery bypass surgery: perioperative predictors and impact on mortality. Braz J Cardiovasc Surg [Internet]. 2015 [acesso em: 30 dez. 2017];30(2):164-72. Disponível em: http://dx.doi.org/10.5935/1678-9741.20140086.

23. Koerich C, Lanzoni GMM, Erdmann AL. Factors associated with mortality in patients undergoing coronary artery bypass grafting. Rev Lat Am Enfermagem [Internet]. 2016 [acesso em: 30 dez. 2017];24:e2748. Disponível em: http://dx.doi.org/10.1590/1518-8345.0708.2748.

24. Dessotte CAM, Figueiredo ML, Rodrigues HF, Furuya RK, Rossi LA, Dantas RAS. Classificação dos pacientes segundo o risco de complicações e mortalidade após cirurgias cardíacas eletivas. Rev. Eletr. Enf. [Internet]. 2016 [acesso em: 30 dez. 2017];18:e1140. Disponível em: https://doi.org/10.5216/ree.v18.37736. 